1972) Rivas Martínez, Izco y Costa, 1973 (Linario clementei-Andryaletum ramosissimae Rivas Goday y Esteve 1972). Pisos termo y mesomediterráneo en ombroclima seco a subhúmedo.

\title{
Comentarios.
}

Dentro de la sección Polium, la subsección Simplicipilosa (series Pumilum y Simplicipilosa) presentan la mayor cantidad de endemismos, tanto en la Península Ibérica como en el Norte de Africa. Son táxones con indumento de tricomas sencillos, carácter más primitivo en la evolución de estos elementos, que el indumento de tricoma ramificado típico de la subsección Polium o Intermedia. Su número cromosómico: $2 \mathrm{n}=26$, es diploide, no siendo frecuentes los poliploides, más característicos de la subsección Polium. La mayoría son táxones edafoendémicos, viven en zonas costeras aisladas de la Península, donde la influencia de las glaciaciones fue escasa y donde se refugió la vegetación esclerófila y las especies térmicas de raíces terciarias. Se pone de manifiesto, por tanto, el carácter paleoendémico en los táxones de la subsección Simplicipilosa, respecto a los táxones del resto de la sección, que tienden a colonizar áreas montanas mediante la poliploidía.

Los endemismos de la Sección Chamaedrys del sureste de España, igualmente con un número cromosómico bajo: $2 \mathrm{n}=32$, viven en zonas áridas montanas; $T$. webbianum o costeras; $T$. intricatum y $T$. fragile, se pueden considerar, dentro de esta Sección, como especies de caracteres primitivos y, posiblemente, al igual que los comentados de la subsección Simplicipilosa, de origen terciario.

(Aceptado para su publicación en junio de 1991)

Dirección de los autores: T. Navarro: Departamento de Biología Vegetal. Facultad de Ciencias. Universidad de Málaga. J.L. Rosúa y J. Molero Mesa: Departamento de Biología Vegetal. Universidad de Granada.

\section{APORTACIONES AL CONOCIMIENTO DEL GÉNERO TEUCRIUM (SECC.POLIUM, SERIE POLIUM) EN MARRUECOS. LAMIACEAE.}

Palabras clave. Teucrium, Marruecos.

Teresa NAVARRO (1) y José Luis ROSÚA(2)

Se proponen dos nuevas combinaciones, Teucrium mesanidum y T. soloitanum, para el género Teucrium L. (Sección Polium Serie Polium), cuyas poblaciones típicas se localizan en la región austro-occidental de Marruecos. Son especies interesantes corológica y florísticamente para el Norte de Africa, por su amplia distribución a lo 
largo de las Cordilleras del Atlas y de la región del Rif, son tambien de interés biosistemático y filogenético para la Serie Polium ya que pueden establecerse relaciones taxonómicas y de vicarianza entre $T$. mesanidum y $T$. lerrouxi, (especie Ibérica de distribución Bética) y entre T. soloitanum y T. murcicum, (especie tambien Ibérica del sureste peninsular y de las Islas Baleares)

Teucrium mesanidum (Litard \& Maire) Navarro y Rosúa stat. et comb. nov.

T. polium subsp. mesanidum Litard \& Maire Mem. Soc. Sci. Nat. Maroc 4:20. 1924 (basionimo).

T. mesanidum (Litard \& Maire) Sauvage \& Vindt Bull. Soc. Sci. Nat. Maroc 35: 289. 1957 (comb. inval.).

T. mesanidum (Litard \& Maire) var. cylindrostachyum (Maire) Sauvage \& Vindt Bull. Soc. Nat. Maroc:289. 1967 (comb. inval)

T. luteum subsp. mesanidum (Litard \& Maire) Greuter \& Burdet Willdenowia 15:80. 1987.

MARRUECOS: Aquelman de Sidi Ali, Pailler, 8-VII-1955, RABAT 61280.

Taxon muy próximo morfológicamente a T. cylindricum Maire, con el que puede confundirse, distinguiendose por el indumento de la inflorescencia pubescente o tomentoso pero nunca densamente algodonoso y por su coloración blanca o amarilla (Sauvage \& Vindt, 1955; 1967). Ecológicamente, T. mesanidum muestra preferencia por los hábitats altomontanos del gran y medio Atlas, observándose una completa convergencia ecológica vicariante a $T$. lerrouxi en las Cordilleras Béticas, y una convergencia adaptativa de la morfológia calicinal, donde los dientes inferiores quedan divididos desde más abajo que los superiores. (Navarro et al., 1990).

Teucrium. soloitanum (Maire) Navarro y Rosúa stat. et comb. nov.

T. capitatum var. majoricum (Rouy) Willk. forma soloitanum Maire Bull. Soc. Hist. Nat. Afric. Nord. 26:445. 1938(comb. inval.)

T. polium subsp. polium var. soloitanum (Maire) Sauvage \& Vindt. Naturalia Monsp. 16:19. 1965.

Typus: Teucrium polium L. var. majoricum (Willk.) Rouy Forma soloitanum, In rupestribus maritimus calcaires ad premon torcum soliam (Cap. Cantin), 5-4-1937, R. Maire. MPU Herb. Afrique du Nord s.n.

MARRUECOS: Cap Cantin, 25-IV-1937, Gatefosse, MPU Herb. Afrique Nord s.n. Beni Soir, dessus de Sidi Said, 3-VI-1918, Maire, MPU Herb Afrique Nord s.n. Rherim Tizougarim, 30-V-1954, Sauvage \& Vindt, RABAT 51284. Cap Safí, 11-XI1955, Sauvage \& VIndt, RABAT 61278.

La interpretación sistemática de este taxon ha sido problemática dada su variabilidad morfológica y su convergencia adaptativa con poblaciones de T. capitatum y T. polium de Marruecos (Sauvage \& Vindt, 1967).

La variación morfológica detectada en su área de distribución ofrece una respuesta biogeográfica similar a la de $T$. murcicum y es relativa a la densidad del 
indumento calicinal y su coloración, blanca o amarilla; forma de los capitulos florales , suboblongos o espiciformes; crenación de los márgenes foliares.y color de la corola, blanca o rosada.

Este taxon del Marruecos occidental presenta una similaridad morfológica muy acentuada (Maire,1938) con otras poblaciones del Norte de Marruecos y de la región del Rif, de corología y ecologías próximas a las de T. murcicum en el sureste de la Península Ibérica y que aparecen identificadas de forma puntual sobre material de herbario por autores clásicos españoles(Sennen, F. 1928-1930. Plantes D’Espagne et du Maroc.) como: T. maroccanum Sennen nom. illeg. y posteriormente revisadas por Sauvage \& Vindt, 1955 como: T. capitatum var. capitatum sub. var. capitatum $(T$. capitatum f.Lazari Sennen); T. capitatum var. corymbiferum sub. var. rufanum (T. maroccanum Sennen) o sub. var. humberti Maire \& Sennen. Un estudio biosistemático detallado de estos táxones, a través de su área de distribución, como el que se está realizando actualmente nos permitirá su actualización sistemática y pondría de manifiesto sus relaciones taxonómicas y biológicas, entre sí y con T. murcicum del sureste de la Península Ibérica y de las Islas Baleares.

\section{BIBLIOGRAFÍA}

SAUVAGE, CH \& J. VINDT. -1955- Synopsis du genre Teucrium sct, Polium au Maroc. Bull. Soc. Nat. Phys. Maroc, 35(4):283-293.

SAUVAGE, CH \& J. VINDT. -1967-Revisión del Herbier de Linstitut Scientifique Chérifien. Notes Critiques. Le Botaniste serie L, 515-436.

MAIRE, R. -1938-Contribution à l'étude de la Flore de l'Afrique du Nord . Bull. Soc. Hist. Nat. Afr. Nord., 29: 445 (1938).

NAVARRO, T , J. L. ROSUA y J. F. MOTA. -1990- Estudio sistemático de los táxones de la Serie Polium, genéro Teucrium L., en las Cordilleras Béticas. Acta Botánica Malacitana, 15:79-91.

(Aceptado para su publicación en septiembre de 1991)

Dirección de los autores: (1): Departamento de Biología Vegetal. Facultad de Ciencias. Univeridad de Málaga. 29080 Málaga. (2): Departamento de Biología Vegetal. Facultad de Ciencias. Universidad de Granada. 18001 Granada.

\section{NOTAS BIOGEOGRÁFICAS Y ECOLÓGICAS SOBRE ALGUNOS TAXONES LITORALES DEL GÉNERO TEUCRIUM L. (LAMIACEAE) EN ANDALUCÍA}

Palabras clave. Teucrium, ecología, Andalucía, España.

Teresa NAVARRO, José Luis ROSÚA y José Ma․ NIETO CALDERA

Los táxones del género Teucrium L., de distribución mediterránea, y cuya corología en la Península Ibérica queda limitada exclusivamente a la banda costera del 